\title{
Effects of Incentive Spirometry on Respiratory Motion in Healthy Subjects Using Cine Breathing Magnetic Resonance Imaging
}

\author{
Toshiaki Kotani, MD, PhD ${ }^{1}$, Tsutomu Akazawa, MD, PhD ${ }^{1}$, Tsuyoshi Sakuma, MD, $\mathrm{PhD}^{1}$, \\ Shigeyuki Nagaya, $\mathbf{R T}^{2}$, Masaru Sonoda, $\mathrm{RT}^{2}$, Yuji Tanaka, $\mathrm{PT}^{3}$, Takehide Katogi, $\mathrm{PT}^{3}$, \\ Tetsuharu Nemoto, MD, $\mathrm{PhD}^{1}$, Shohei Minami, $\mathrm{MD}, \mathrm{PhD}^{1}$
}

Departments of ${ }^{1}$ Orthopedic Surgery, ${ }^{2}$ Radiology, and ${ }^{3}$ Rehabilitation, Seirei Sakura Citizen Hospital, Chiba, Japan

Objective To investigate the effectiveness of incentive spirometry on respiratory motion in healthy subjects using cine breathing magnetic resonance imaging (MRI).

Methods Ten non-smoking healthy subjects without any history of respiratory disease were studied. Subjects were asked to perform pulmonary training using incentive spirometry every day for two weeks. To assess the effectiveness of this training, pulmonary function tests and cine breathing MRI were performed before starting pulmonary training and two weeks after its completion.

Results After training, there were significant improvements in vital capacity (VC) from $3.58 \pm 0.8 \mathrm{~L}$ to $3.74 \pm 0.8 \mathrm{~L}$ and in \%VC from 107.4 \pm 10.8 to $112.1 \pm 8.2$. Significant changes were observed in the right diaphragm motion, right chest wall motion, and left chest wall motion, which were increased from $55.7 \pm 9.6 \mathrm{~mm}$ to $63.4 \pm 10.2 \mathrm{~mm}$, from 15.6 $\pm 6.1 \mathrm{~mm}$ to $23.4 \pm 10.4 \mathrm{~mm}$, and from $16.3 \pm 7.6 \mathrm{~mm}$ to $22.0 \pm 9.8 \mathrm{~mm}$, respectively.

Conclusion Two weeks of training using incentive spirometry provided improvements in pulmonary function and respiratory motion, which suggested that incentive spirometry may be a useful preoperative modality for improving pulmonary function during the perioperative period.

Keywords Spirometry, Respiratory function tests, Magnetic resonance imaging

\section{INTRODUCTION}

Received May 13, 2014; Accepted October 8, 2014

Corresponding author: Toshiaki Kotani

Department of Orthopedic Surgery, Seirei Sakura Citizen Hospital, 2-362 Ebaradai Sakura-shi, Chiba 285-8765, Japan

Tel: +81-43-486-1151, Fax: +81-43-486-8696, E-mail: tkotani@wa2.so-net. ne.jp

(ㄷ) This is an open-access article distributed under the terms of the Creative Commons Attribution Non-Commercial License (http://creativecommons. org/licenses/by-nc/4.0) which permits unrestricted noncommercial use, distribution, and reproduction in any medium, provided the original work is properly cited.

Copyright () 2015 by Korean Academy of Rehabilitation Medicine
Currently, incentive spirometry (IS) is a widely used modality for preventing or treating postoperative atelectasis [1]. Several studies have evaluated the effectiveness of IS; however, their results are conflicting [2-6]. Moreover, although respiratory physiotherapy using IS can have a mechanical effect on the respiratory motion of the chest wall and diaphragm, this aspect is unclear.

There are some reports on dynamic analysis of chest wall and diaphragm motions using cine magnetic reso- 
nance imaging (MRI) [7,8]. Although cine breathing MRI (BMRI) has led to a better understanding of respiratory function [9-11], the use of cine BMRI of the chest wall and diaphragm has not been reported with regard to the effectiveness of IS. Therefore, the purpose of this study was to investigate the effectiveness of IS on respiratory motion in healthy subjects using cine BMRI.

\section{MATERIALS AND METHODS}

Ten non-smoking healthy subjects without any history of respiratory disease were studied: 7 women and 3 men, whose mean age was 31.2 \pm 6.1 years (range, 21-40 years). This study was approved by the ethics committee of Seirei Sakura Citizen Hospital, and all participants were informed about the study procedures before they gave their written consent to participate in this study.

\section{Incentive spirometry training program}

For IS, a flow-oriented device (Triflo II, Teleflex/Hudson RCI, Temecula, CA, USA; and Respirex, DHD Medical Products, New York, NY, USA) was used. The training menu using IS was based on the techniques that are commonly used preoperatively for orthopedic surgery in our hospital. Five deep breaths were performed via an incentive spirometer in order to achieve sustained maximal inspirations and expirations. Education was given about how to use the device, and subjects were instructed to take five sets of five sustained maximal inspirations and expirations two times a day; i.e., each patient took a total of 50 maximal inspirations and expirations daily using an incentive spirometer. Subjects were asked to perform their pulmonary training every day for two weeks. To assess the effectiveness of this training, pulmonary function tests (PFTs) and cine BMRI were performed before starting pulmonary training and two weeks after its completion.

\section{Pulmonary function tests}

All PFTs were performed using the same computerized spirometer. These tests were performed while the patients were standing, and each measurement was repeated three times. The best of 3 measures was taken as the final measure. The results for standard respiratory function tests-vital capacity (VC), \%VC, forced expiratory volume in 1 second (FEV1), and the ratio of FEV1 to FVC
(FEV1\%)-were expressed as mean \pm standard deviation.

\section{Cine BMRI}

Cine BMRI was performed using a 1.5-T MR system with a whole body coil (Signa Twin Speed 1.5T; GE Medical Systems, Milwaukee, WI, USA). For localization, a T1weighted coronal anatomic image of a patient's thorax with breath-holding after full expiration was used. Fast Imaging Employing Steady-state Acquisition (FIESTA) sequences were used in cine BMRI. Sequential parameters were TR/TE $=4.0 / 1.2 \mathrm{~ms}$; flip angle, $30^{\circ}$; slice thickness, 5 $\mathrm{mm}$; matrix, $160 \times 160$. Scans were performed in the supine position to estimate vital-capacity breathing without breath-holding. Respiratory rates were 3 to 6 breaths in 30 seconds. Kinematic motions of the thorax were recorded every 0.5 seconds for 30 seconds in the sagittal and axial planes. Sagittal images were acquired at the middle of both lungs. Axial images were acquired at the level of the center of the T10 vertebra. Each MR image was sent to a personal computer and analysis was performed using the National Institute of Health Image software (http://rsb. info.nih.gov/nih-image/). The chest wall and diaphragm motions were animated and evaluated visually using a cine-loop view of the sequential MR images.

\section{BMRI variables}

Images at maximum full inspiration and minimum full expiration were identified in each dynamic sequence. In the sagittal and axial planes, respiratory motions of the right and left thorax were measured separately. In the midsagittal planes, a line was drawn tangent to the lung apex and perpendicular to the vertical MRI frame. On the full inspiratory and full expiratory images, the distances from this line to the diaphragm at its highest point were defined as the maximum dimension (Di) and minimum dimension (De), respectively. Diaphragm motion was defined as the difference between Di and De (Fig. 1). Chest wall dimensions in the anteroposterior diameter of the thorax in the axial planes were also measured (Fig. 2). The results for BMRI variables were expressed as mean \pm standard deviation.

Paired t-tests were used for statistical comparisons, with $\mathrm{p}<0.05$ being considered statistically significant. In our clinical data, 1) there were no outliers; 2) there were no large differences between trimmed means and general means; 3) Shapiro-Wilk tests showed that all our data 
(A)

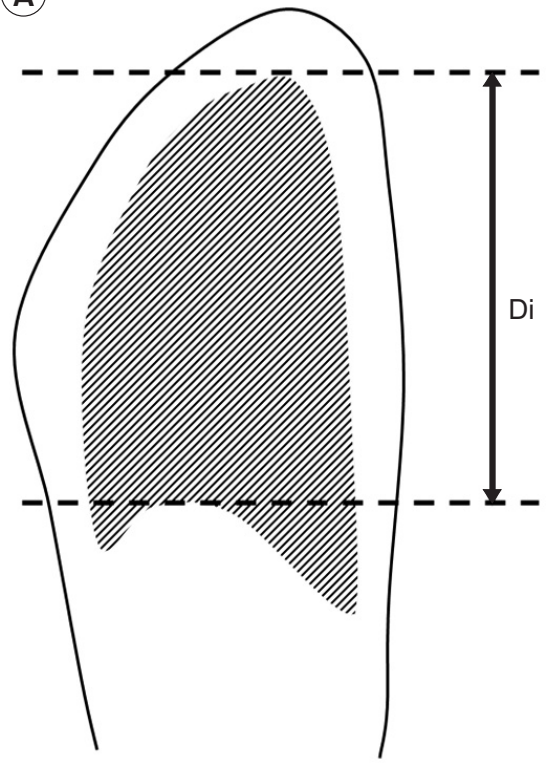

(A)

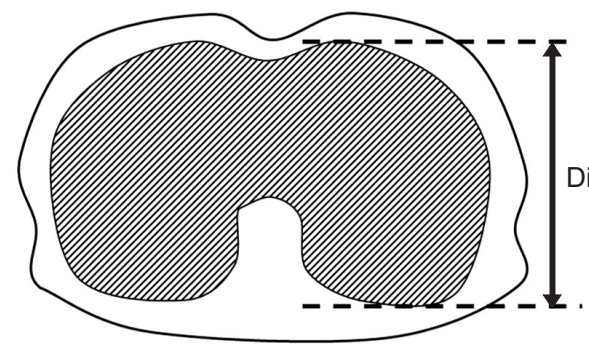

B)

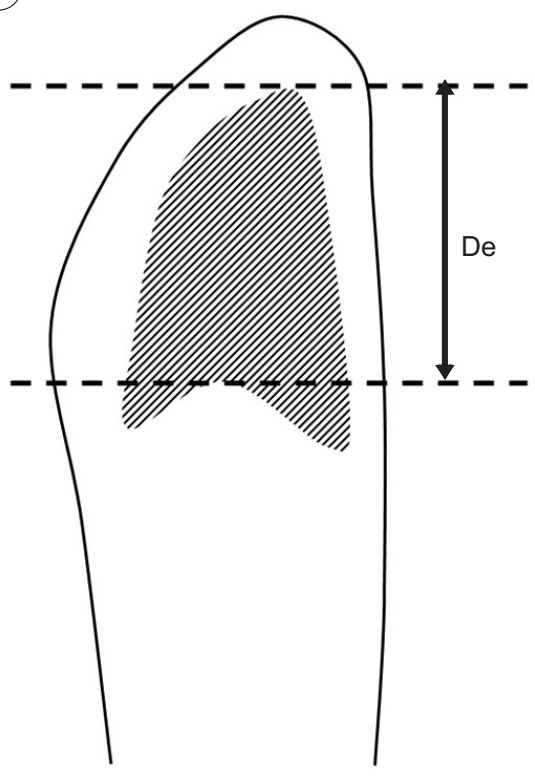

Fig. 1. Right midsagittal maximal inspiratory (A) and expiratory (B) images. We drew a line tangent to the lung apex and perpendicular to the vertical magnetic resonance imaging frame. The distances from this line to the diaphragm at its highest point are defined as the maximum dimension (Di) or minimum dimension (De), respectively.

(B)

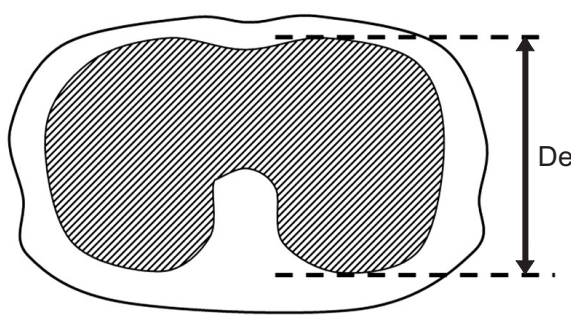

Fig. 2. Lower axial (at the level of the center of the T10 vertebra) maximal inspiratory (A) and expiratory (B) images.

were normally distributed, except for the data for postrehabilitation right diaphragm motion and pre-rehabilitation left chest wall motion; and 4) quantile-quantile plots did not show large differences. Although our clinical data were derived from only 10 subjects, for the above mentioned reasons we judged that paired t-tests were sufficiently robust based on the central limit theorem. Statistical analysis was performed using SPSS Statistics ver. 20 (IBM Corporation, Armonk, NY, USA).

\section{RESULTS}

An entire sequence was completed within 30 minutes. The spatial resolution of the individual images and the temporal resolution of the constructed cine-loops were considered to be of diagnostic quality for all of the subjects. Significant improvements in VC and \%VC from $3.6 \pm 0.8 \mathrm{~L}$ to $3.7 \pm 0.8 \mathrm{~L}$ and from $107.4 \pm 10.8$ to $112.1 \pm 8.2$,
Table 1. Absolute values and percent predicted values of pulmonary function tests pre- and post-training

\begin{tabular}{lccc}
\hline & Pre-training & Post-training & p-value \\
\hline VC (L) & $3.6 \pm 0.8$ & $3.7 \pm 0.8$ & $0.023^{*}$ \\
\%VC & $107.4 \pm 10.8$ & $112.1 \pm 8.2$ & $0.028^{*}$ \\
FEV1 (L) & $3.1 \pm 0.5$ & $3.2 \pm 0.6$ & 0.110 \\
FEV1\% & $87.9 \pm 4.3$ & $87.7 \pm 5.7$ & 0.871 \\
\hline
\end{tabular}

VC, vital capacity; FEV1, forced expiratory volume in 1 second.

*Significant result, $\mathrm{p}<0.05$.

respectively, were observed after training. FEV1 and FEV1\% values did not change significantly (Table 1). Left diaphragm motion was not significantly altered $(\mathrm{p}=0.057)$, which was increased from $54.3 \pm 12.2 \mathrm{~mm}$ to $60.4 \pm 7.7 \mathrm{~mm}$. In contrast, significant changes were observed in right diaphragm motion, right chest wall motion, and left chest 
wall motion, which were increased from $55.7 \pm 9.6 \mathrm{~mm}$ to $63.4 \pm 10.2 \mathrm{~mm}$, from $15.6 \pm 6.1 \mathrm{~mm}$ to $23.4 \pm 10.4 \mathrm{~mm}$, and from 16.3 $\pm 7.6 \mathrm{~mm}$ to $22.0 \pm 9.8 \mathrm{~mm}$, respectively (Table 2 ).

This subject was a 24-year-old healthy female. After two weeks of training using IS, her VC, \%VC, FEV1, and FEV1\% were increased from $3.38 \mathrm{~L}, 108.3 \%, 3.20 \mathrm{~L}$, and $91.4 \%$ to $3.62 \mathrm{~L}, 116.0 \%, 3.34 \mathrm{~L}$, and $94.9 \%$, respectively. Her pre- and post-training chest wall and diaphragm motions were animated and evaluated visually using a cineloop view of the sequential MR images. Pre- and posttraining, right midsagittal, maximal inspiratory images

Table 2. Diaphragm and chest wall motions before and after training (unit, $\mathrm{mm}$ )

\begin{tabular}{lccc}
\hline & Pre-training & Post-training & p-value \\
\hline Rt. diaphragm & $55.7 \pm 9.6$ & $63.4 \pm 10.2$ & $0.031^{*}$ \\
Lt. diaphragm & $54.3 \pm 12.2$ & $60.4 \pm 7.7$ & 0.057 \\
Rt. chest wall & $15.6 \pm 6.1$ & $23.4 \pm 10.4$ & $0.009^{*}$ \\
Lt. chest wall & $16.3 \pm 7.6$ & $22.0 \pm 9.8$ & $0.019^{*}$ \\
\hline
\end{tabular}

Rt., right; Lt., left.

*Significant result, $\mathrm{p}<0.05$. showed increased chest wall and diaphragm motion after training (Fig. 3A, B).

\section{DISCUSSION}

An incentive spirometer is a device designed to achieve and sustain maximal inspiration. It is easy to use and provides the patient with visual feedback on air flow and volume. Its use results in a prolonged phase of effective inspiration, more controlled flow, and greater enthusiasm to practice [12]. However, the efficacy of IS remains controversial [5,13]. Thomas and McIntosh [5] reported that IS and deep breathing exercises appeared to be more effective than no physical therapy intervention in preventing postoperative pulmonary complications. However, Schwieger et al. [14] showed that on comparing the effects of IS with a control group, there were no betweengroup changes in lung function. In a systematic review, Carvalho et al. [15] concluded that there was no evidence to support the use of IS in the management of surgical patients. A linear relationship between chest wall motion and lung volume changes has been reported $[16,17]$.
(A)
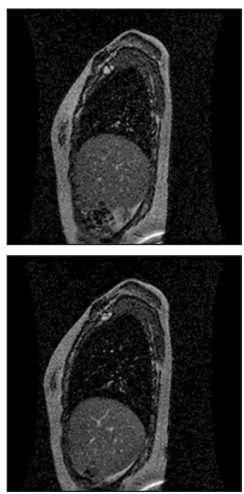

(B)
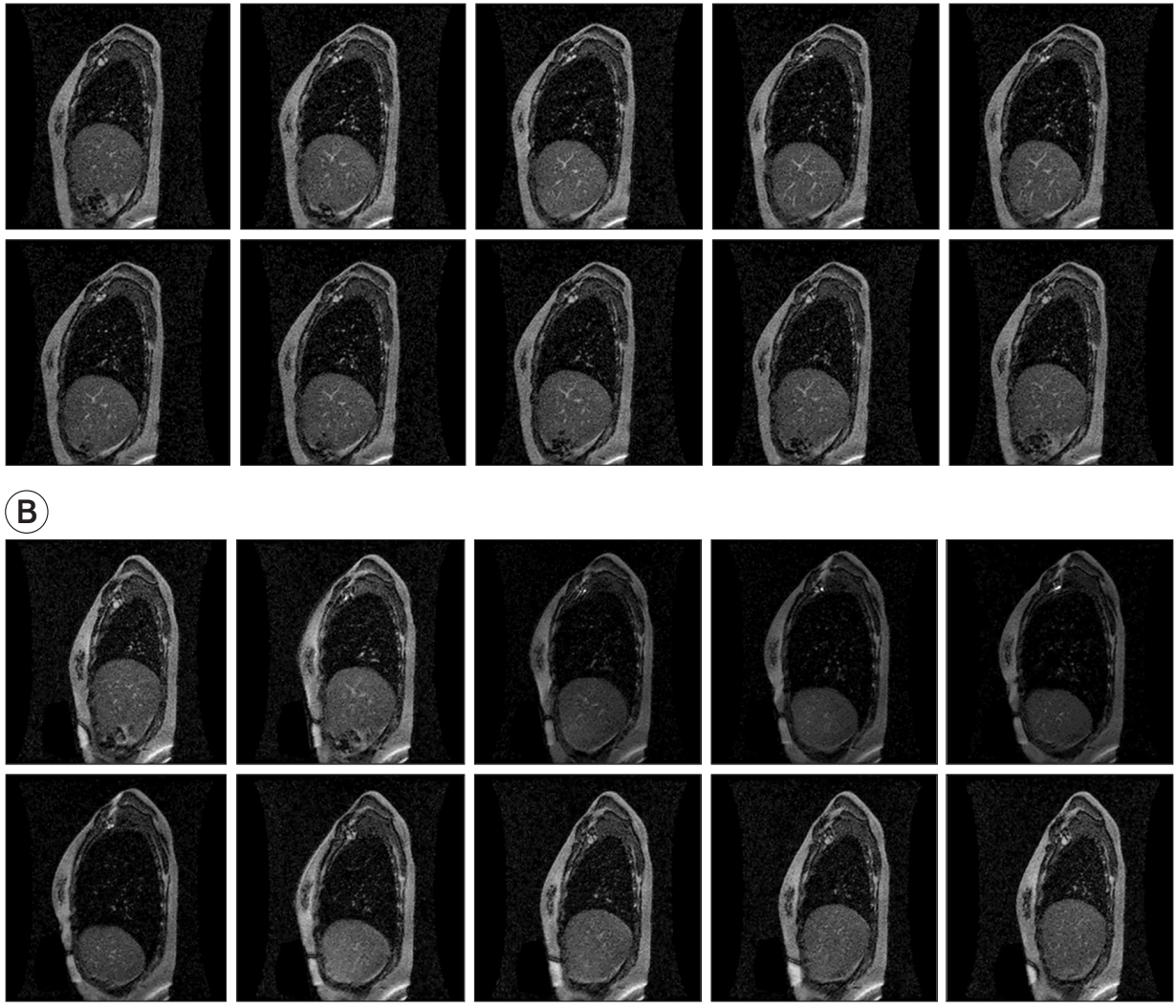

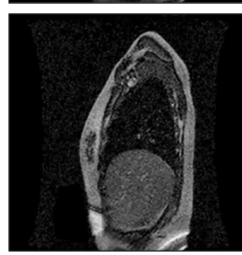

Fig. 3. Pre-training (A) and posttraining (B) right midsagittal maximal inspiratory images in a healthy subject. Chest wall and diaphragm motions are increased after training. 
Only a few studies have focused on physiological aspects, such as thoracoabdominal motion, while using IS $[18,19]$. In these studies, respiratory plethysmography was used to assess the breathing patterns and thoracoabdominal motions during breathing exercises using IS. However, the authors did not mention the effects after some period of respiratory training using IS.

In the current study, we found that two weeks of pulmonary physiotherapy using IS provided improvements in pulmonary function and respiratory motion, which suggested that IS may be a useful preoperative modality for improving pulmonary function during the perioperative period. Among our motion measurements, only left diaphragm motion did not improve significantly ( $p=0.057)$, which may be due to the small sample size. The present results are compatible with those reported by Thomas and McIntosh [5]. However, the findings of previous studies have not always been in agreement. These discrepancies could be due to differences in the amount of physiotherapy using IS and differences in patient selection. To the best of our knowledge, this is the first report to study the effects of pulmonary training with IS on respiratory motion using cine BMRI. The present results also demonstrated excellent feasibility of cine BMRI for assessing diaphragmatic and chest wall motions directly and visually. Quantitative measures enable us to assess focal respiratory motions before and after respiratory training. BMRI enables us to understand diaphragm and chest wall motions and to plan pulmonary training that is appropriate for respiratory condition of each patient.

There were some limitations to the present study. Our sample size was small. This type of study needs to be repeated in a larger population. Also, we only investigated the healthy subjects. In some departments, such as the orthopedic department, IS is also used for preoperative patients who have normal lung function. Our results showed that two weeks of preoperative training with IS for patients who had normal lung function could be useful and it can be recommended. Furthermore, its effectiveness may depend on patient selection; thus, its effect in different types of patients should be investigated.

In conclusion, using cine BMRI, we evaluated respiratory motions before and after pulmonary training using IS. Although this study was only a preliminary trial, our results suggest that two weeks of respiratory training using IS is useful for improving respiratory motion and pulmonary function. Further studies are needed to investigate the efficacy of pulmonary training using IS in different types of patients using cine BMRI.

\section{CONFLICT OF INTEREST}

No potential conflict of interest relevant to this article was reported.

\section{REFERENCES}

1. O'Donohue WJ Jr. Prevention and treatment of postoperative atelectasis. Can it and will it be adequately studied? Chest 1985;87:1-2.

2. Chuter TA, Weissman C, Starker PM, Gump FE. Effect of incentive spirometry on diaphragmatic function after surgery. Surgery 1989;105:488-93.

3. Gosselink R, Schrever K, Cops P, Witvrouwen H, De Leyn $\mathrm{P}$, Troosters $\mathrm{T}$, et al. Incentive spirometry does not enhance recovery after thoracic surgery. Crit Care Med 2000;28:679-83.

4. Overend TJ, Anderson CM, Lucy SD, Bhatia C, Jonsson BI, Timmermans C. The effect of incentive spirometry on postoperative pulmonary complications: a systematic review. Chest 2001;120:971-8.

5. Thomas JA, McIntosh JM. Are incentive spirometry, intermittent positive pressure breathing, and deep breathing exercises effective in the prevention of postoperative pulmonary complications after upper abdominal surgery? A systematic overview and metaanalysis. Phys Ther 1994;74:3-10.

6. Weindler J, Kiefer RT. The efficacy of postoperative incentive spirometry is influenced by the device-specific imposed work of breathing. Chest 2001;119:1858-64.

7. Biederer J, Hintze C, Fabel M, Dinkel J. Magnetic resonance imaging and computed tomography of respiratory mechanics. J Magn Reson Imaging 2010;32:138897.

8. Fabel M, Wintersperger BJ, Dietrich O, Eichinger M, Fink C, Puderbach M, et al. MRI of respiratory dynamics with 2D steady-state free-precession and 2D gradient echo sequences at 1.5 and 3 Tesla: an observer preference study. Eur Radiol 2009;19:391-9.

9. Gierada DS, Curtin JJ, Erickson SJ, Prost RW, Strandt JA, Goodman LR. Diaphragmatic motion: fast gradient-recalled-echo MR imaging in healthy subjects. 
Radiology 1995;194:879-84.

10. Kondo T, Kobayashi I, Taguchi Y, Ohta Y, Yanagimachi N. A dynamic analysis of chest wall motions with MRI in healthy young subjects. Respirology 2000;5:19-25.

11. Kotani T, Minami S, Takahashi K, Isobe K, Nakata Y, Takaso M, et al. An analysis of chest wall and diaphragm motions in patients with idiopathic scoliosis using dynamic breathing MRI. Spine (Phila Pa 1976) 2004;29:298-302.

12. Westwood K, Griffin M, Roberts K, Williams M, Yoong $\mathrm{K}$, Digger T. Incentive spirometry decreases respiratory complications following major abdominal surgery. Surgeon 2007;5:339-42.

13. Doyle RL. Assessing and modifying the risk of postoperative pulmonary complications. Chest 1999;115(5 Suppl):77S-81S.

14. Schwieger I, Gamulin Z, Forster A, Meyer P, Gemperle M, Suter PM. Absence of benefit of incentive spirometry in low-risk patients undergoing elective cholecystectomy: a controlled randomized study. Chest 1986;89:652-6.

15. Carvalho CR, Paisani DM, Lunardi AC. Incentive spi- rometry in major surgeries: a systematic review. Rev Bras Fisioter 2011;15:343-50.

16. Cohen E, Mier A, Heywood P, Murphy K, Boultbee J, Guz A. Excursion-volume relation of the right hemidiaphragm measured by ultrasonography and respiratory airflow measurements. Thorax 1994;49:885-9.

17. Gauthier AP, Verbanck S, Estenne M, Segebarth C, Macklem PT, Paiva M. Three-dimensional reconstruction of the in vivo human diaphragm shape at different lung volumes. J Appl Physiol (1985) 1994;76:495506.

18. Parreira VF, Tomich GM, Britto RR, Sampaio RF. Assessment of tidal volume and thoracoabdominal motion using volume and flow-oriented incentive spirometers in healthy subjects. Braz J Med Biol Res 2005;38:1105-12.

19. Tomich GM, França DC, Diorio AC, Britto RR, Sampaio RF, Parreira VF. Breathing pattern, thoracoabdominal motion and muscular activity during three breathing exercises. Braz J Med Biol Res 2007;40:140917. 